\title{
Clinical influence of maternal serum homocysteine, folate and vitamin B12 in the development of pre- eclampsia
}

https://doi.org/10.1515/pteridines-2019-0006

received November 12, 2018; accepted January 28, 2019.

Abstract: Background: The aim of this study was to investigate the correlation between maternal serum homocysteine (Hcy), folate, vitamin B12 (VitB12) and the development of pre-eclampsia (PE).

Methods: Seventy-eight normal pregnant women (without hypertension and proteinuria during their pregnancy (control group)), 66 cases of gestational hypertension (GH group ) and 82 cases of pre-eclampsia (PE group, with 56 cases of mild disease and 26 cases of severe disease) were include in this study. The maternal serum Hcy, folate and VitB12 level of the included cases were examined between 11 to 13 weeks gestation and compared between each group.

Results: The serum levels of VitB12 were significantly different between the control, GH and PE groups ( $p<0.05)$. The serum levels of Hcy in the PE group were significantly higher than those of the control group $(\mathrm{p}<0.05)$. However, the serum levels of folate in the PE group were significantly lower than those of control group $(p<0.05)$. Significant statistical differences in the maternal serum Hcy and folate were found between mild and severe PE patient groups $(p<0.05)$. A significant correlation was found between maternal serum Hcy and VitB12 ( $r=-0.34$, $\mathrm{p}=0.001$ ).

Conclusion: Hcy, folate and VitB12 may play an important role in the development of PE and could be potential serological biomarkers for early PE diagnosis.

Keywords: Homocysteine; Folate; vitamin B12; preeclampsia; gestational hypertension

\footnotetext{
*Corresponding author: Xu Zeng, Department of ICU, Lishui Central Hospital(Lishui Hospital of Zhejiang Unviersity; $5^{\text {TH }}$ Affiliated Hospital of Wenzhou Medical University), 323000 PR China, E-mail: 58177434@qq.com

Hongyan Zhao: Department of Obstetrics, Lishui Central Hospital (Lishui Hospital of Zhejiang Unviersity; $5^{\mathrm{TH}}$ Affiliated Hospital of Wenzhou Medical University), 323000 PR China
}

\section{Introduction}

Epidemiological studies have shown that preeclampsia (PE) complicates about 3\% of pregnancies and all hypertensive disorders affect about five to $10 \%$ of pregnancies [1]. Gestational hypertension (GH) or pregnancy-induced hypertension (PIH) is the development of hypertension during the pregnancy without the presence of protein in the urine or other signs of PE. Another indication of $\mathrm{GH}$ includes blood pressure greater than 140/90 mm Hg. PE is defined as a disorder of pregnancy characterized by the onset of high blood pressure and often a significant amount of protein in the urine. Whether GH and PE are different stages of the same disease of is still up for debate [2].

Hcy is an important intermediate product in methionine metabolism. Folic acid and VitB12 are important intermediate products for homocysteine synthesis [3]. It had been proven that elevated serum Hcy is a risk factor for both cardiovascular disease and vasculopathy $[4,5]$. Although the exact pathogenesis of PE remains unclear, some studies have demonstrated that the development of PE is associated with general endothelial dysfunction, including vasoconstriction and end-organ ischemia [6]. Studies have also shown that endothelial cell injury or dysfunction is central to the development of PE [7]. Elevated plasma Hcy levels can lead to vascular endothelial damage, but the correlation between plasma Hcy levels and PE development is not consistently reported in the literature [8]. Under the action of folate acting as a methyl donor and VitB12 acting as a coenzyme, Hcy can be re-methylated into methionine. Therefore, serum folate and VitB12 are involved in the regulation of serum Hcy concentrations. Implicit in this generalized endothelial dysfunction may be an imbalance of angiogenic and anti-angiogenic factors. The relationship between serum Hcy and PE has been discussed previously in different publications [9-10]. However, the gradual changes of maternal Hcy, folate and VitB12 from GH to severe PE has rarely been reported. In our present work, we evaluated 
the correlation of maternal serum Hcy, folate, VitB12 and the development of PE in order to evaluate their role in the development of PE.

\section{Material and methods}

Patients: Seventy-eight normal pregnant women (without hypertension and proteinuria during the pregnancy (control group)), 66 cases of gestational hypertension (GH group) and 82 cases of pre-eclampsia (PE group, with 56 cases of mild disease and other 26 cases of severe disease) were included in this study. The maternal serum Hcy, folate and VitB12 levels of the included cases were examined between 11 to 13 weeks gestation and compared between each group.

Inclusion criteria: (1) Written informed consent was obtained from all patients. (2) The GH and PE were clinically confirmed. The PE was diagnosed if blood pressure was continuously elevated after 20 weeks of gestation with $>=140 / 90 \mathrm{~mm} \mathrm{Hg}$ together with proteinuria ( $>=300 \mathrm{mg}$ of protein over 24 hours, or a random dipstick urine determination of $>=1+$ protein or $>=30 \mathrm{mg} / \mathrm{dL}$ ). Blood pressure was elevated on at least 2 occasions more than 6 hours apart. (3) All cases included in the study were singleton pregnancies. (4) All the cases had follow-up to delivery. (5) Cases in the control group without hypertension and proteinuria during the pregnancy.

Exclusion criteria: (1) Chronic disease history before pregnancy such as hypertension, diabetes mellitus and thyroid disease. (2) History of recurrent spontaneous abortion. (3) HIV positive or other sexually transmitted diseases. (4) Multiple pregnancy.

Informed consent: Written informed consent was obtained from all patients according to the declaration of Helsinki.

Ethical approval: The research related to human use has been complied with all the relevant national regulations, institutional policies and in accordance the tenets of the Helsinki Declaration, and has been approved by the ethics committee of Lishui Central Hospital (Lishui Hospital of Zhejiang Unviersity; 5TH Affiliated Hospital of Wenzhou Medical University).

\section{Maternal Serum Hcy, Folate and Vitamin B12 Detection}

Serum level of Hcy was measured by the Hcy Assay Kit of Enzymatic Method(Biological\& Technological Inc, Wuhan, China). Serum folic acid was tested by the ARCHITECT Folic Acid Reagent Kit (Abbott, Ireland). Serum VitB12 in was examined by the ARCHITECT B12 Reagent Kit (Abbott, Ireland). All the experimental procedures were carried out according to the manufacturer's instructions.

\section{Statistical methods}

The data was analyzed by SPSS 17.0 statistical software (SPSS, Inc., Chicago, IL, USA) and the figures was drawn by Graph pad Prism 7.0 (http://www.graphpad.com/ scientific-software/prism/). The maternal serum Hcy, folate and VitB12 was expressed by $\bar{x} \pm s$ and compared by t-test between each of the groups. The correlation between maternal serum Hcy, folate and VitB12 were analyzed by a Pearson correlation test. $\mathrm{P}<0.05$ meant that there was a statistical significance.

\section{Results}

\section{Baseline demographic characteristics of the included cases}

The general characteristics of the included cases are demonstrated in Table 1. There was no statistical significance in the BMI, primiparous, smoking history, drinking history, age of husband and education level $\left(\mathrm{p}_{\text {all }}>0.05\right)$. However, the age of control group was significantly lower compared to the PE group $(p<0.05)$ while the age of $\mathrm{GH}$ and $\mathrm{PE}$ groups were not statistically significance $(p>0.05)$. This suggests that age maybe a potential risk factor for PE.

\section{Maternal Serum Homocysteine, Folate and vitamin B12}

The mean levels of serum Hcy, folate and VitB12 were showed in Table 2. The serum levels of VitB12 were significantly higher in control group compared to that of GH and PE groups $(p<0.05)$. The serum level of Hcy in PE 
Table 1: Baseline demographic characteristics of the included cases.

\begin{tabular}{|c|c|c|c|c|c|c|}
\hline Characteristics & Control $(n=78)$ & GH $(n=66)$ & $\mathrm{PE}(\mathrm{n}=82)$ & p-value & & \\
\hline & & & & Control vs GH & Control vs PE & GH vs $P E$ \\
\hline Age (y) & $28.6 \pm 3.6$ & $29.1 \pm 4.0$ & $30.3 \pm 3.8$ & 0.43 & 0.004 & 0.07 \\
\hline BMI $\left(\mathrm{kg} / \mathrm{m}^{2}\right)$ & $21.6 \pm 2.4$ & $22.1 \pm 2.7$ & $22.4 \pm 3.0$ & 0.24 & 0.07 & 0.53 \\
\hline Primiparous [n(\%)] & & & & 0.72 & 0.56 & 0.36 \\
\hline Yes & $62(79.5)$ & $54(81.8)$ & $62(75.6)$ & & & \\
\hline NO & $16(20.5)$ & $12(18.2)$ & $20(24.4)$ & & & \\
\hline Smoking history [n(\%)] & & & & 0.70 & 0.48 & 0.29 \\
\hline Yes & $6(7.7)$ & $4(6.1)$ & 9(10.9) & & & \\
\hline NO & 72(92.3) & 62(93.9) & 73(89.1) & & & \\
\hline Drinking history & & & & 0.56 & 0.68 & 0.33 \\
\hline Yes & $16(20.5)$ & $11(16.7)$ & $19(23.2)$ & & & \\
\hline NO & $62(79.5)$ & $55(83.3)$ & $63(76.8)$ & & & \\
\hline Age of husband (y) & $31.2 \pm 6.6$ & $30.8 \pm 5.8$ & $32.6 \pm 6.1$ & 0.70 & 0.17 & 0.07 \\
\hline Education level [n(\%)] & & & & 0.49 & 0.88 & 0.40 \\
\hline Primary or secondary education & $40(51.3)$ & $30(45.5)$ & 43(52.4) & & & \\
\hline Higher education or above & $38(48.7)$ & $36(54.5)$ & $39(47.6)$ & & & \\
\hline
\end{tabular}

Table 2: Maternal Serum Homocysteine, Folate and vitamin B12 comparison between different groups.

\begin{tabular}{|c|c|c|c|c|c|c|}
\hline Item & Control(n=78) & $\mathrm{GH}(n=66)$ & $\mathrm{PE}(\mathrm{n}=\mathbf{8 2})$ & p-value & & \\
\hline & & & & Control vs GH & Control vs PE & GH vs $P E$ \\
\hline $\mathrm{Hcy}(\mu \mathrm{mol} / \mathrm{L})$ & $7.25 \pm 1.37$ & $7.59 \pm 2.24$ & $8.29 \pm 2.78$ & 0.26 & 0.003 & 0.10 \\
\hline Folate(nmol/L) & $30.80 \pm 4.54$ & $29.32 \pm 4.57$ & $28.30 \pm 4.90$ & 0.05 & 0.001 & 0.19 \\
\hline VitB12 (pmol/L) & $387.60 \pm 45.76$ & $369.40 \pm 50.11$ & $336.30 \pm 43.70$ & 0.02 & $<0.0001$ & $<0.0001$ \\
\hline
\end{tabular}

group was significantly higher than those of control group $(p<0.05)$. However, the serum level folate in PE group was significantly lower than those of control group $(p<0.0)$ (Figure 1).

\section{Maternal Serum Homocysteine, Folate and vitamin B12 comparison between mild and severe PE patients}

Notably, the maternal serum Hcy and folate levels were found to be significantly different between mild and severe PE patients $(\mathrm{p}<0.05)$. However, the maternal serum VitB12 was not statistically different between mild and severe PE patients ( $p>0.05)$ (Table 3).
Table 3: Maternal Serum Homocysteine, Folate and vitamin B12 level in PE groups.

\begin{tabular}{lllll}
\hline Item & Mild PE & Severe PE & t & p-value \\
\hline Hcy $(\mu \mathrm{mol} / \mathrm{L})$ & $7.74 \pm 2.60$ & $9.49 \pm 2.82$ & 2.75 & $\mathbf{0 . 0 0 7}$ \\
Folate $(\mathrm{nmol} / \mathrm{L})$ & $29.27 \pm 4.57$ & $23.23 \pm 5.03$ & 2.72 & $\mathbf{0 . 0 0 8}$ \\
VitB12 $(\mathrm{pmol} / \mathrm{L})$ & $342.40 \pm 34.64$ & $323.10 \pm 57.29$ & 1.90 & 0.061 \\
\hline
\end{tabular}

\section{Correlation between maternal serum Hcy, folate and vitamin B12}

The association between maternal serum Hcy, folate and VitB12 were tested by Pearson correlation test. A significant correlation was found between maternal serum Hcy and VitB12 ( $\mathrm{r}=-0.34, \mathrm{p}=0.001)$ (Figure 2). 
A

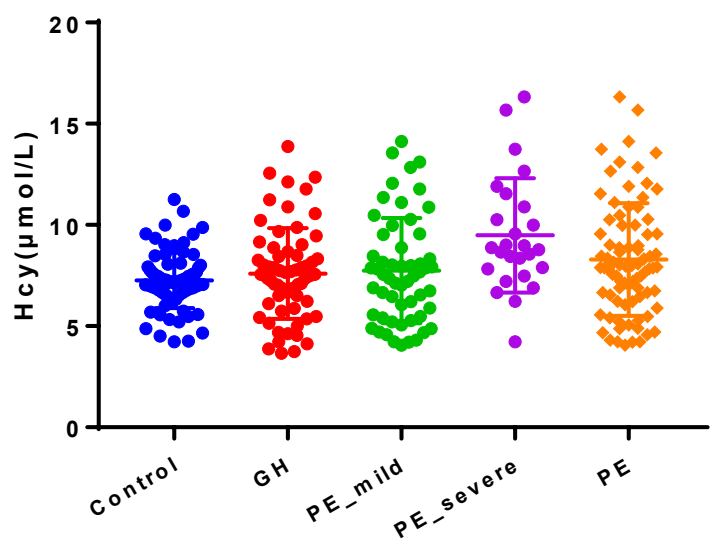

C

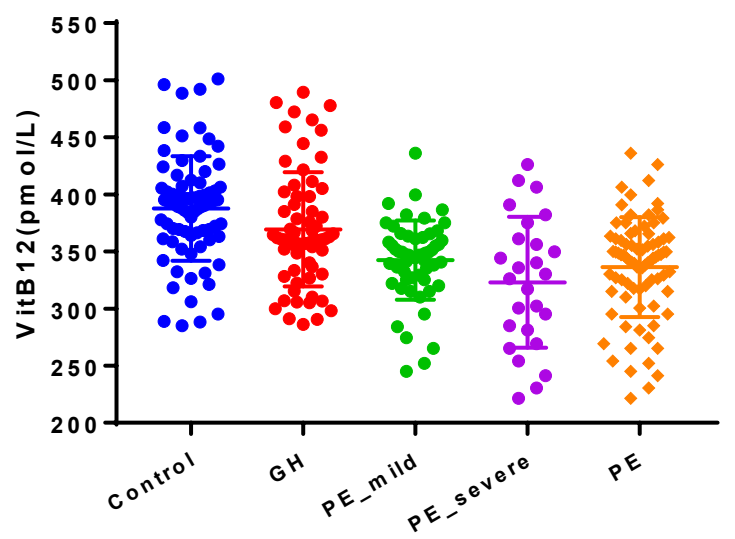

B

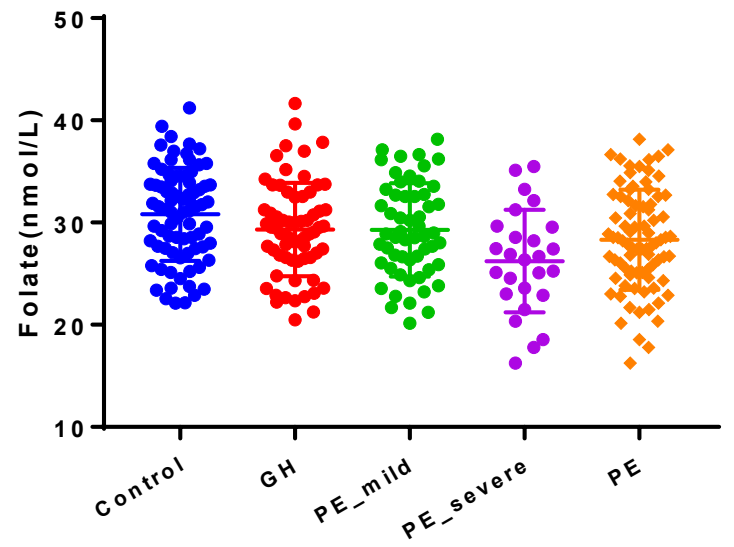

Figure 1: Scatter plot of serum Hcy, folate and ViB12 level (A: serum Hcy distribution; B: serum folate distribution; C: serum ViB12 distribution).

\section{Discussion}

$\mathrm{PE}$ is one of the most widely diagnosed pregnancy-related complications with the incidence rate of $3 \%$ of all the pregnancies [1]. However, the exact pathogenesis has remained unclear. Studies have shown that endothelial cell injury or dysfunction are important factors in the development of PE [11]. Elevated plasma Hcy level can lead to vascular endothelial damage, but the correlation between plasma Hcy levels and the risk of development of PE has not been clearly demonstrated in previous publications [12-15]. Hcy promotes the production of reactive oxygen species, also known as free radicals, which results in oxidative stress and damage of vascular endothelial cells, thus causing systemic arterial spasm and other pathophysiological manifestations, which conforms to the pathogenesis of PE. Studies have found that the plasma Hcy levels in PE patients is about 1.7 to
1.8 times higher than that of normal pregnant women [16]. Acilmis et al. (2011) have shown that the occurrence of hypertensive disorders in pregnancy is related to an increase in the plasma Hcy levels in pregnant women and that the level of Hcy is related to the severity of the disease [17]. In our present work, we found that the serum levels of Hcy in PE group was significantly higher than those of control group ( $p<0.05)$. Additionally, the serum level in severe PE patients was significantly higher than those of mild PE patients. This indicated that with the development of PE, the serum Hcy had gradually increased, thereby suggesting that Hcy may play an important role in the development of PE. However, the exact mechanism of action of Hcy in the development of PE is unclear.

Folate and VitB12 are essential for DNA synthesis and cell growth, which were also involved in Hcy metabolism. A lack of folate and VitB12 may lead to an increase in serum levels of Hcy. Previous publications have shown 
A

$r=-0.16, p=0.14$

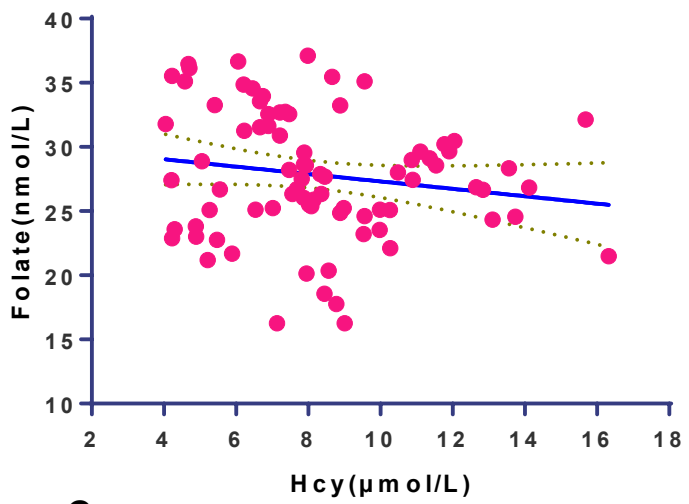

C

$r=-0.03, p=0.82$

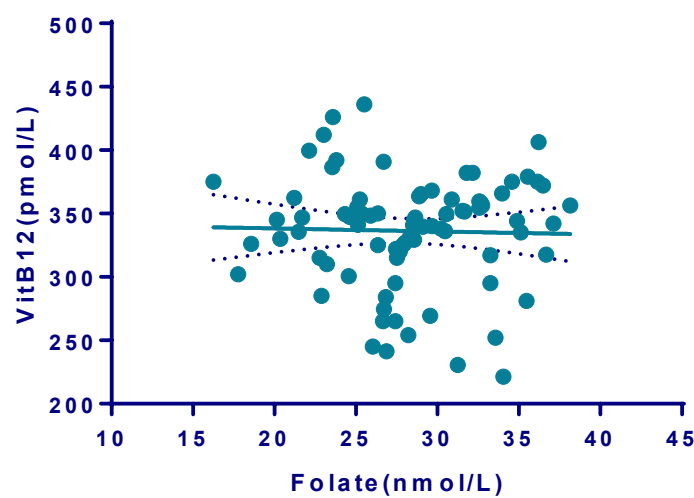

Figure 2: Pearson correlation analysis for serum Hcy, folate and VitB12.

that an increase of dietary folic acid can lead to a reduction in the serum levels of Hcy along with a reduced risk for developing PE [18]. However, other publications have indicated that this increase in folate dietary intake is not associated with a decrease in the serum Hcy levels [14]. In our study, we found that the serum level of folate and VitB12 were significantly lower in the PE group compared to the GH group. We also found that in patients with severe $\mathrm{PE}$, the serum levels of folate and VitB12 were significantly lower than those of mild PE group. This data suggests that decreased serum levels of folate and VitB12 are linked to the development of PE which was in accordance with the previously reported studies.

\section{B}

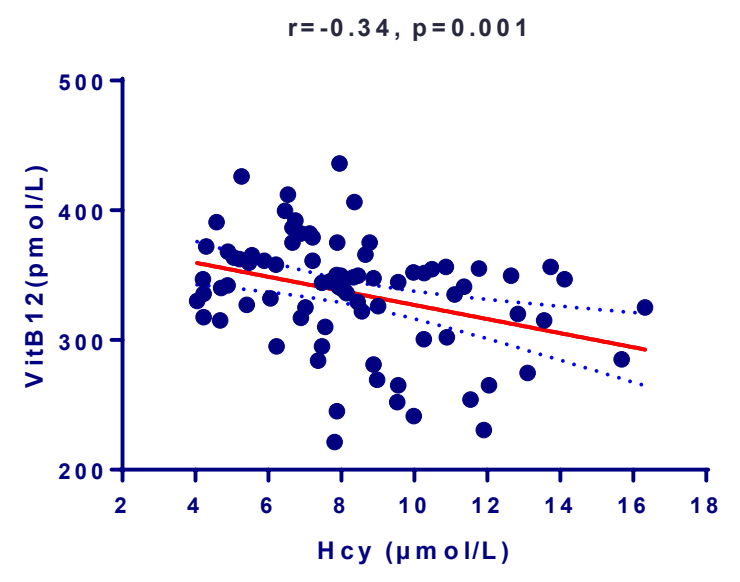




\section{References}

1. Hutcheon JA, Lisonkova S, Joseph KS. Epidemiology of preeclampsia and the other hypertensive disorders of pregnancy. Best Pract Res Clin Obstet Gynaecol 2011;25:391-403.

2. Uguz F. Is There Any Association Between Use of Antidepressants and Preeclampsia or Gestational Hypertension?: A Systematic Review of Current Studies. J Clin Psychopharmacol 2017;37:72-77.

3. Obeid R, Herrmann W. Homocysteine, folic acid and vitamin $B 12$ in relation to pre- and postnatal health aspects. Clinical chemistry and laboratory medicine 2005;43:1052-7.

4. Cybulska B, Klosiewicz-Latoszek L. Homocysteine-is it still an important risk factor for cardiovascular disease. Kardiologia polska 2015;73:1092-6.

5. Blom HJ, Smulders Y. Overview of homocysteine and folate metabolism. With special references to cardiovascular disease and neural tube defects. J Inherit Metab Dis 2011;34:75-81.

6. Mustafa R, Ahmed S, Gupta A, Venuto RC. A comprehensive review of hypertension in pregnancy. J Pregnancy 2012;2012:105918.

7. Roberts JM, Taylor RN, Musci TJ, Rodgers GM, Hubel CA, McLaughlin MK. [Pre-eclampsia: a disorders of the endothelial cells?]. Gynakologe 1992;25:2-6.

8. Mao D, Che J, Li K, Han S, Yue Q, Zhu L, et al. Association of homocysteine, asymmetric dimethylarginine, and nitric oxide with preeclampsia. Arch Gynecol Obstet 2010;282:371-5.

9. Guven MA, Coskun A, Ertas IE, Aral M, Zencirci B, Oksuz H. Association of maternal serum CRP, IL-6, TNF-alpha, homocysteine, folic acid and vitamin B12 levels with the severity of preeclampsia and fetal birth weight. Hypertension in pregnancy 2009;28:190-200.

10. Hasanzadeh M, Ayatollahi H, Farzadnia M, Ayati S, Khoob MK. Elevated plasma total homocysteine in preeclampsia. Saudi Med J 2008;29:875-8.

11. Wu C, Yuan J, Sui R, Li S, Sun J. Tanshinone II-A is protective against human umbilical vein endothelial cell injury after exposure to serum from preeclampsia patients. Gynecol Obstet Invest 2014;78:101-8.

12. Malek-khosravi S, Kaboudi M, Kaboudi B, Atefi G. Plasma homocysteine concentrations and insulin resistance in preeclampsia. Hypertension in pregnancy 2009;28:13-22.

13. Braekke K, Ueland PM, Harsem NK, Karlsen A, Blomhoff R, Staff AC. Homocysteine, cysteine, and related metabolites in maternal and fetal plasma in preeclampsia. Pediatr Res 2007;62:319-24.

14. Makedos G, Papanicolaou A, Hitoglou A, Kalogiannidis I, Makedos A, Vrazioti V, et al. Homocysteine, folic acid and B12 serum levels in pregnancy complicated with preeclampsia. Arch Gynecol Obstet 2007;275:121-4.

15. Baksu A, Taskin M, Goker N, Baksu B, Uluocak A. Plasma homocysteine in late pregnancies complicated with preeclampsia and in newborns. Am J Perinatol 2006;23:31-5.

16. Cotter AM, Molloy AM, Scott JM, Daly SF. Elevated plasma homocysteine in early pregnancy: a risk factor for the development of nonsevere preeclampsia. Am J Obstet Gynecol 2003;189:391-4; discussion 394-6.
17. Acilmis YG, Dikensoy E, Kutlar Al, Balat O, Cebesoy FB, Ozturk E, et al. Homocysteine, folic acid and vitamin B12 levels in maternal and umbilical cord plasma and homocysteine levels in placenta in pregnant women with pre-eclampsia. J Obstet Gynaecol Res 2011;37:45-50.

18. Wen SW, Chen XK, Rodger M, White RR, Yang Q, Smith GN, et al. Folic acid supplementation in early second trimester and the risk of preeclampsia. Am J Obstet Gynecol 2008;198:45.e1-7. 